\title{
The association between single nucleotide polymorphisms in TNFAIP3 and serological activity markers of rheumatoid arthritis in the Chinese Han population
}

\author{
XINGANG ZHANG ${ }^{l}$,WEI $L^{2}$, XINPENG ZHANG ${ }^{3}, Y U N$ GUO $^{l}$, LI JIANG ${ }^{l}$, XIAOLI ZHANG ${ }^{l}$, \\ XIAOFEI WANG ${ }^{l}$ \\ ${ }^{1}$ The $1^{\text {st }}$ Department of Rheumatology, Shengjing Hospital of China Medical University, Shenyang, Liaoning Province, China \\ ${ }^{2}$ Editorial Department of Chinese Pediatric Emergency Medicine, Shenyang, Liaoning Province, China \\ ${ }^{3}$ Department of Neurosurgery, The Fourth People's Hospital of Shenyang City, Shenyang, Liaoning Province, China
}

\begin{abstract}
Aim of the study: To identify TNFAIP3 SNP genotypes that confer a high risk of active rheumatoid arthritis $(R A)$ through serological activity markers in the Han population of Chinese RA patients.

Material and methods: A hospital-based untreated case study of 344 RA patients was conducted. Three polymorphisms were determined by the TaqMan SNP genotyping method. Serological activity markers were tested by regular detection means.

Results: 1. ANOVA indicated a near significant difference in IgG between rs2230926 and rs13207033/rs10499194 ( $p=0.062)$. A significant difference was identified in C3 between rs13207033 and rs2230926/rs10499194 ( $p=0.047)$. 2. According to pairwise comparisons, the IgG levels among the patients with TT or TG genotypes at rs2230926 were significantly higher than among the patients with $G G(p=0.019$ or 0.023 , respectively). The C3 levels among the GG or GA patients at rs 13207033 were significantly lower than among the patients with $A A(p=0.01$ for both). 3. According to the Pearson $\chi^{2}$ test and $O R s$, the RF-positive rate among the patients with $G G+T G$ at rs 2230926 was lower than among the TT patients $(O R=0.36,95 \%$ CI: 0.14-0.96; $p=0.034)$. The RF-positive rate for the TG patients was lower than for the TT patients $(O R=0.38,95 \%$ CI: $0.14-1.00 ; p=0.051)$. The anti-CCP antibody-positive rate among the $A A+G A$ patients at rs 13207033 was lower than among the GG patients $(O R=0.37,95 \%$ CI: 0.13-1.04; $p=0.053)$.

Conclusions: The TT or TG genotypes at rs2230926 and the AA or GG genotypes at rs 13207033 may be high-risk genotypes for RA as indicated by serological activity indicators in the Chinese Han population.
\end{abstract}

Key words: TNFAIP3 polymorphisms, rheumatoid arthritis, serological activity markers.

(Centr Eur J Immunol 2013; 38 (4): 409-415)

\section{Introduction}

Single nucleotide polymorphisms (SNPs) describe the variation in DNA sequences at the single base-pair level that occurs among members of the same species. Single nucleotide polymorphisms account for many phenotypic differences among individuals and populations. Several gene variants have been identified which predispose individuals to rheumatoid arthritis (RA) [1,2]. Among Caucasians, the tumor necrosis factor (TNF)- $\alpha$ induced protein 3 gene (TNFAIP3) has been identified as a genetic risk factor for RA [3, 4]. TNFAIP3 encodes the A20 protein, which functions in ubiquitin editing. A20 is necessary for the ter- mination of TNF-induced signaling and negatively regulates the nuclear factor (NF)- $\kappa \mathrm{B}$ signaling pathway; both of these pathways are actively involved in the development of RA [5, 6]. Mice deficient in A20 may develop severe inflammation, including inflammation of the joints [7]. Therefore, mutations in TNFAIP3 may increase the risk of RA development. We previously examined three SNPs in TNFAIP3 and reported that two of these (rs10499194 and rs2230926) were related to the occurrence of RA in the Chinese Han population. However, few reports have identified correlations between all three SNP loci in TNFAIP3 (rs10499194, rs2230926, and rs13207033) and severity of RA among the Chinese Han population. 
Rheumatoid arthritis is a systemic disease characterized by chronic destructive joint lesions. The primary manifestation of RA is symmetric small joint polyarthritis, which may be accompanied by extra-articular manifestations such as fever, anemia, subcutaneous nodules, and enlarged lymph nodes. Active RA may be identified by its characteristic symptoms, serology, and imaging. Serological indicators are direct, objective, and interrelated. We used serology in the Han population of Chinese RA patients to identify TNFAIP3 SNP genotypes that confer a high risk for active RA. This study lays the basis for future gene therapy against high-risk genes.

\section{Material and methods}

\section{Study subjects and data collection}

Data were collected from 344 patients (mean age 54.0 years, $83.7 \%$ of the patients were women) with confirmed diagnosis of RA untreated who visited the Rheumatology and Immunology wards at the Shengjing Hospital of China Medical University between July 2010 and June 2011. All patients met the diagnostic criteria of the American College of Rheumatology (ACR 1987) [8]. Patients with infections, primary diabetes, primary hypertension, primary hyperlipidemia, primary cardio-cerebrovascular diseases, primary liver and renal diseases, primary thrombotic diseases, primary platelet disorders, and acute and chronic hemorrhages caused by other factors were excluded from the study. This study was approved by the Ethics Committee of the Shengjing Hospital of China Medical University, all subjects provided written informed consent and the ethics committee approved this consent procedure. All participants were of the Chinese Han nationality without kinship. Each subject provided a fasting venous blood sample $(2 \mathrm{ml})$ in the morning.

\section{Selection of SNP loci}

Previous reports have described the following RA susceptibility loci within and near TNFAIP3: rs10499194, rs2230926, and rs6920220 [9-11]. However, only SNPs with minor allele frequencies $(\mathrm{MAF}) \geq 0.1$ are regarded as appropriate for correlation or lineage studies [12]. HapMap data from the Chinese population indicated that the MAF of rs6920220 was $<0.001$. A study investigating the Korean population also suggested that the rs6920220 mutation was too rare $(\mathrm{MAF}<0.01)$ to evaluate it for associations [13]. In addition, we reviewed the correlation between SNPs at various TNFAIP3 loci and RA in the Asian population. Therefore, we selected rs10499194, rs2230926, and rs 13207033 for the evaluation of SNPs.

\section{DNA isolation and genotyping}

Genomic DNA samples were isolated by the phenolchloroform method. Single nucleotide polymorphisms were analyzed by TaqMan assays. For the selected SNPs, predesigned TaqMan SNP genotyping assays were used. Primers and probes were synthesized by Shanghai Generay Biotechnology Co, Ltd. (P.R. China) (Table 1). The sequences of probes and primers are listed in Table 1. Fluorescence was detected using an ABI Prism 7500 FAST Sequence Detection System (Applied Biosystems). Genotyping assessment was performed on $>98 \%$ of the samples for each of the polymorphisms genotyped. A $10 \%$ masked random sample of patients was tested twice by different persons, and the results were found to be in agreement for all of the duplicate sets.

\section{Selection of serological activity indicators}

White blood cell (WBC), hemoglobin (HB), platelet (PLT), immunoglobulin (Ig) M, IgG, IgA, fibrinogen (FIB), D-dimer (DD), complement (C) 3, C4, erythrocyte

Table 1. Sequence of primers and probes of SNP

\begin{tabular}{lcr}
\hline SNP & Name & Sequence \\
\hline rs2230926 & forward primer & GATTTGAGAGACTCCAGTTGCCA \\
\cline { 2 - 3 } & reverse primer & GCGTTCAGGACACAGACTTGG \\
\cline { 2 - 3 } rs13207033 & probe 1 & FAM-AGCGTGCTGAACAGCGCCTTC-TAMRA \\
\cline { 2 - 3 } & forward primer & HEX-AGCGTGCTGCACAGCGCCT-TAMRA \\
\cline { 2 - 3 } & reverse primer & gCACAATgAAAgAgAgAgAAgTagAATAAT \\
\hline rs10499194 & probe 1 2 & FAM-TACAgATTTCACTTTCCCT-MgB \\
\hline & forward primer & HEX-CTACAgATTTTACTTTCC-MGB \\
\cline { 2 - 3 } & reverse primer & gCTATCAgTTTCATTACCTAAgAAATAgAgAC \\
\hline & probe 1 & CAA Cgg ATA AgC AAT CAg ACC A \\
\hline
\end{tabular}


sedimentation rate (ESR), C-reactive protein (CRP), rheumatoid factor $(\mathrm{RF})$, anticyclic citrullinated peptide (anti-CCP) antibody, and anti-cardiolipin antibody (ACA) were selected to evaluate RA disease activity.

\section{Testing of serological activity indicators}

1. The electrical impedance method was used to measure WBC, HB, and PLT.

2. Immunoturbidimetry was used to examine $\operatorname{IgM}, \operatorname{IgG}$, IgA, C3, C4, CRP, RF, FIB, and DD.

3. Enzyme-linked immunosorbent assay (ELISA) was used to test samples for anti-CCP antibody and ACA.

4. Westergren's method was used to measure the ESR.

\section{Quality control}

Based on the serological findings, investigators were divided into a routine group and an immunological group ( 3 investigators/group). Two members of each group simultaneously collected data from the same indicators, and the third member checked for data consistency.

In each experiment, negative controls and replicate samples were used for quality control. Ten percent of samples were randomly selected for blinded repeated testing.

\section{Statistical analysis}

All statistical analyses were performed with SPSS (v17.0), if not otherwise specified. Samples were grouped by SNP genotype. Then, SNP indicators were compared for differences between SNP genotypes, including pairwise differences. Analysis of variance (ANOVA) was used to evaluate measurements of WBC, HB, PLT, IgM, IgG, IgA, FIB, DD, C3, C4, and ESR. The Pearson $\chi^{2}$ test was used for categorical data, including CRP, RF, anti-CCP antibody, and ACA. Clinical characteristics were compared across genotypes, and a corresponding odds ratio (OR) and $95 \%$ confidence interval $(95 \% \mathrm{CI})$ were calculated. Statistical significance was defined as $p<0.05$.

\section{Results}

1. ANOVA (Table 2) indicated a near significant difference in IgG between rs2230926 and rs13207033/ rs10499194 $(p=0.062)$. The other indicators were not significantly different in this comparison. We identified a significant difference in $\mathrm{C} 3$ between rs 13207033 and rs2230926/rs10499194 ( $p=0.047)$. The other indicators were not significantly different in this comparison. No significant differences were detected among the indicators when rs10499194 and rs2230926/rs13207033 were evaluated.

2. According to pairwise comparisons (Table 3), the IgG levels among the patients with TT or TG genotypes at rs2230926 were significantly higher than among the patients with GG ( $p=0.019$ or 0.023 , respectively). The C3 levels among the GG or GA patients at rs13207033 were significantly lower than among the patients with AA $(P=0.01$ for both $)$.

3. According to the Pearson $\chi^{2}$ test and Ors (Table 4), the RF-positive rate among the patients with GG+TG at rs2230926 was lower than among the TT patients (OR $=0.36,95 \%$ CI: 0.14-0.96; $p=0.034)$. The RF-positive rate for the TG patients was lower than for the TT patients $(\mathrm{OR}=0.38,95 \% \mathrm{CI}: 0.14-1.00 ; p=0.051)$. No significant differences were detected regarding CRP, anti-CCP antibody, or ACA among various genotypes at rs2230926. The anti-CCP antibody-positive rate among the AA+GA patients at rs 13207033 was lower than among the GG patients (OR $=0.37,95 \%$ CI: 0.13 $1.04 ; p=0.053)$. There were no significant differences regarding CRP, RF, or ACA among different genotypes at rs13207033.

\section{Discussion}

The correlation research between SNPs and disease activity has emerged as an area of avid research. Rheumatoid arthritis is a common autoimmune disease for which genetic factors can contribute highly deleterious or protective ef-

Table 2. The ANOVA results of the measurement data from different genotypes in RA patients

\begin{tabular}{|c|c|c|c|c|c|c|c|c|c|c|c|c|}
\hline \multirow[t]{2}{*}{ Genotype } & \multicolumn{2}{|c|}{ WBC } & \multicolumn{2}{|c|}{ HB } & \multicolumn{2}{|c|}{ PLT } & \multicolumn{2}{|c|}{ IgM } & \multicolumn{2}{|c|}{ IgG } & \multicolumn{2}{|c|}{ IgA } \\
\hline & $F$ value & $p$ value & $F$ value & $p$ value & $F$ value & $p$ value & $F$ value & $p$ value & $F$ value & $p$ value & $F$ value & $p$ value \\
\hline rs2230926 & 2.017 & 0.135 & 0.735 & 0.48 & 1.377 & 0.254 & 0.47 & 0.626 & 2.808 & 0.062 & 0.985 & 0.375 \\
\hline rs 13207033 & 0.329 & 0.72 & 1.509 & 0.223 & 0.988 & 0.373 & 0.063 & 0.939 & 0.461 & 0.631 & 0.428 & 0.652 \\
\hline \multirow[t]{3}{*}{ rs10499194 } & 2.825 & 0.094 & 0.748 & 0.388 & 0.243 & 0.623 & 0.857 & 0.356 & 0.105 & 0.746 & 0.526 & 0.469 \\
\hline & \multicolumn{2}{|c|}{ FIB } & \multicolumn{2}{|c|}{ DD } & \multicolumn{2}{|c|}{$\mathrm{C3}$} & \multicolumn{2}{|c|}{$\mathrm{C} 4$} & \multicolumn{2}{|c|}{ ESR } & & \\
\hline & $F$ value & $p$ value & $F$ value & $p$ value & $F$ value & $p$ value & $F$ value & $p$ value & $F$ value & $p$ value & & \\
\hline rs 2230926 & 1.459 & 0.235 & 0.187 & 0.83 & 0.328 & 0.721 & 1.24 & 0.291 & 1.105 & 0.332 & & \\
\hline rs 13207033 & 0.208 & 0.812 & 0.067 & 0.935 & 3.086 & 0.047 & 1.768 & 0.173 & 0.353 & 0.703 & & \\
\hline rs 10499194 & 0.381 & 0.537 & 0.565 & 0.453 & 0.156 & 0.693 & 0.445 & 0.505 & 0.259 & 0.611 & & \\
\hline
\end{tabular}


Table 3. The pairwise comparison results of the measurement data from different genotypes at rs 2230926 and rs 13207033 in RA patients

\begin{tabular}{|c|c|c|c|c|c|c|c|c|c|c|}
\hline \multirow{2}{*}{$\begin{array}{l}\text { Dependent } \\
\text { variable }\end{array}$} & \multirow[t]{2}{*}{ (I) } & \multirow[t]{2}{*}{$(\mathbf{J})$} & \multirow[t]{2}{*}{ Sig. } & \multicolumn{2}{|c|}{ 95\% Confidence Interval } & \multirow[t]{2}{*}{ (I) } & \multirow[t]{2}{*}{$(\mathbf{J})$} & \multirow[t]{2}{*}{ Sig. } & \multicolumn{2}{|c|}{ 95\% Confidence Interval } \\
\hline & & & & Lower Bound & Upper Bound & & & & Lower Bound & Jpper Bound \\
\hline \multicolumn{6}{|c|}{ rs2230926 } & \multicolumn{5}{|c|}{ rs13207033 } \\
\hline \multirow[t]{2}{*}{ Z-score (WBC) } & \multirow[t]{2}{*}{$\mathrm{TT}$} & TG & 0.05 & -0.5667 & 0.00545 & \multirow[t]{2}{*}{ GG } & GA & 0.599 & -0.3595 & 0.20784 \\
\hline & & GG & 0.63 & -1.0475 & 1.73636 & & AA & 0.551 & -0.9746 & 1.82384 \\
\hline \multirow[t]{2}{*}{ Z-score (HB) } & \multirow[t]{2}{*}{$\mathrm{TT}$} & TG & 0.502 & -0.38532 & 0.189055 & \multirow[t]{2}{*}{ GG } & GA & 0.1671 & -0.48164 & 0.083693 \\
\hline & & GG & 0.326 & -0.69841 & 2.096428 & & AA & 0.3193 & -0.68728 & 2.10101 \\
\hline \multirow[t]{2}{*}{ Z-score (PLT) } & \multirow[t]{2}{*}{$\mathrm{TT}$} & TG & 0.163 & -0.49014 & 0.083098 & \multirow[t]{2}{*}{ GG } & GA & 0.4472 & -0.17359 & 0.392642 \\
\hline & & GG & 0.399 & -0.79552 & 1.993812 & & $\mathrm{AA}$ & 0.2274 & -0.53794 & 2.254803 \\
\hline \multirow[t]{2}{*}{ Z-score (IgM) } & \multirow[t]{2}{*}{ TT } & TG & 0.911 & -0.29048 & 0.325559 & \multirow[t]{2}{*}{ GG } & GA & 0.9433 & -0.32986 & 0.306833 \\
\hline & & GG & 0.335 & -0.71358 & 2.090098 & & AA & 0.7299 & -1.15746 & 1.650348 \\
\hline \multirow[t]{2}{*}{ Z-score (IgG) } & \multirow[t]{2}{*}{ TT } & TG & 0.818 & -0.26959 & 0.341008 & \multirow[t]{2}{*}{ GG } & GA & 0.9368 & -0.33066 & 0.305055 \\
\hline & & GG & 0.019 & 0.280162 & 3.059054 & & AA & 0.3382 & -2.08476 & 0.718766 \\
\hline \multirow[t]{2}{*}{ Z-score (IgA) } & \multirow[t]{2}{*}{$\mathrm{TT}$} & TG & 0.337 & -0.15731 & 0.457524 & \multirow[t]{2}{*}{ GG } & GA & 0.8124 & -0.27955 & 0.356246 \\
\hline & & GG & 0.289 & -0.64412 & 2.154043 & & AA & 0.3776 & -2.03117 & 0.772706 \\
\hline \multirow[t]{2}{*}{ Z-score (FIB) } & \multirow[t]{2}{*}{$\mathrm{TT}$} & TG & 0.5137 & -0.7645 & 0.3831 & GG & GA & 0.772 & -0.38925 & 0.28936 \\
\hline & & GG & 0.8416 & -3.1122 & 2.5378 & & AA & 0.5746 & -1.00529 & 1.807635 \\
\hline Z-score (DD) & $\mathrm{TT}$ & TG & 0.9788 & -187.7864 & 182.7654 & GG & GA & 0.7434 & -0.396 & 0.283052 \\
\hline & & GG & 0.535 & -624.0112 & 1199.7488 & & AA & 0.8818 & -1.3011 & 1.513635 \\
\hline Z-score (C3) & $\mathrm{TT}$ & TG & 0.536 & -0.05545 & 0.10636 & GG & GA & 0.7013 & -0.06598 & 0.097948 \\
\hline & & GG & 0.584 & -0.26317 & 0.466083 & & AA & 0.0142 & 0.091423 & 0.812883 \\
\hline Z-score (C4) & $\mathrm{TT}$ & TG & 0.175 & -0.00986 & 0.054002 & GG & GA & 0.2501 & -0.01353 & 0.051725 \\
\hline & & GG & 0.396 & -0.08165 & 0.205791 & & $\mathrm{AA}$ & 0.1265 & -0.03176 & 0.255065 \\
\hline Z-score (ESR) & $\mathrm{TT}$ & TG & 0.676 & -7.00318 & 4.548915 & GG & GA & 0.6794 & -4.57347 & 7.007772 \\
\hline & & GG & 0.16 & -7.67485 & 46.46587 & & $\mathrm{AA}$ & 0.4748 & -37.0064 & 17.27091 \\
\hline
\end{tabular}

fects. For this reason, RA can exhibit a wide range of activities across patients with similar basic characteristics (e.g. lifestyle, gender, geographical region, and age of onset).

Because RA is a systemic vascular inflammatory disease, the numbers of WBCs can increase significantly in RA patients with severe inflammatory changes. Anemia is one of the most common extra-articular manifestations of RA. Other than the reported association between anemia and the use of non-steroidal anti-inflammatory drugs and immunosuppressants, anemia correlates with RA activity in most RA patients. Platelets are involved in systemic vascular inflammatory changes [14]. We found no differences in WBC, HB, or PLT among various SNP genotypes at three TNFAIP3 loci.

Our study identified significantly elevated $\mathrm{IgG}$ levels among the patients with TT or TG at rs2230926 compared with the GG patients ( $p=0.019$ or 0.023 , respectively). Therefore, harboring the TT or TG genotype may be a ge- netic risk factor, whereas the GG genotype may be a protective factor.

Fibrinogen is a component of the acute-phase protein response [15] that contributes to inflammation mainly by interacting with WBCs via cell-surface receptors. Recent studies involving FIB have focused on coagulation and cardiovascular diseases, especially coronary atherosclerosis $[16,17]$. We did not find any correlation between FIB and TNFAIP3 SNPs, possibly because of different disease activities at study enrollment. However, Arvidson et al. [18] suggested that FIB was related to RA activity, and Yildirim et al. [19] reported that FIB was more closely related to RA activity than the ESR. D-dimer is a specific marker of secondary fibrinolysis. This study identified no difference in DD among various TNFAIP3 SNPs. Recently, studies of autoimmune diseases have increased. This was especially true for RA-naïve patients, suggesting an association with inflammatory changes. 
Table 4. The Pearson Chi square test results of the count data from different genotypes in RA patients

\begin{tabular}{|c|c|c|c|c|c|c|c|c|c|c|c|c|c|}
\hline Genotype & & CRP(+) & CRP(-) & OR & Lower & Upper & $p$ value & $\mathbf{R F}(+)$ & $\mathbf{R F}(-)$ & OR & Lower & Upper & $p$ value \\
\hline \multirow[t]{4}{*}{ rs2230926 } & TT & 197 & 42 & \multicolumn{4}{|c|}{ reference } & 174 & 50 & \multicolumn{4}{|c|}{ reference } \\
\hline & TG & 47 & 8 & 0.8 & 0.35 & 1.81 & 0.591 & 46 & 5 & 0.38 & 0.14 & 1 & 0.051 \\
\hline & GG & 1 & 1 & 4.69 & 0.29 & 76.9 & 0.278 & 2 & 0 & - & - & - & - \\
\hline & $\mathrm{GG}+\mathrm{TG}$ & 48 & 9 & 0.88 & 0.4 & 1.96 & 0.749 & 48 & 5 & 0.36 & 0.14 & 0.96 & 0.034 \\
\hline \multirow[t]{4}{*}{ rs13207033 } & GG & 202 & 39 & \multicolumn{4}{|c|}{ reference } & 179 & 50 & \multicolumn{4}{|c|}{ reference } \\
\hline & GA & 41 & 12 & 1.52 & 0.73 & 3.14 & 0.263 & 41 & 5 & 0.44 & 0.16 & 1.16 & 0.097 \\
\hline & AA & 2 & 0 & - & - & - & - & 2 & 0 & - & - & - & - \\
\hline & $\mathrm{AA}+\mathrm{GA}$ & 43 & 12 & 1.45 & 0.7 & 2.99 & 0.318 & 43 & 5 & 0.42 & 0.16 & 1.11 & 0.071 \\
\hline \multirow[t]{3}{*}{ rs10499194 } & $\mathrm{CC}$ & 229 & 47 & \multicolumn{4}{|c|}{ reference } & 209 & 51 & \multicolumn{4}{|c|}{ reference } \\
\hline & $\mathrm{CT}$ & 16 & 4 & 1.22 & 0.39 & 3.8 & 0.734 & 13 & 4 & 1.26 & 0.39 & 4.03 & 0.696 \\
\hline & & $\mathrm{CCP}(+)$ & $\mathrm{CCP}(-)$ & OR & Lower & Upper & $p$-value & $\operatorname{ACA}(+)$ & $\operatorname{ACA}(-)$ & OR & Lower & Upper & $p$ value \\
\hline \multirow[t]{4}{*}{ rs2230926 } & TT & 86 & 30 & \multicolumn{4}{|c|}{ reference } & 30 & 37 & \multicolumn{4}{|c|}{ reference } \\
\hline & TG & 20 & 8 & 1.15 & 0.46 & 2.87 & 0.77 & 5 & 7 & 1.14 & 0.33 & 3.94 & 0.842 \\
\hline & GG & 1 & 0 & - & - & - & - & 0 & 1 & - & - & - & - \\
\hline & GG+TG & 21 & 8 & 1.08 & 0.44 & 2.73 & 0.85 & 5 & 8 & 1.3 & 0.38 & 4.38 & 0.674 \\
\hline \multirow[t]{4}{*}{ rs 13207033} & GG & 76 & 33 & \multicolumn{4}{|c|}{ reference } & 30 & 33 & \multicolumn{4}{|c|}{ reference } \\
\hline & GA & 30 & 5 & 0.38 & 0.13 & 1.08 & 0.069 & 5 & 12 & 2.18 & 0.69 & 6.92 & 0.185 \\
\hline & AA & 1 & 0 & - & - & - & - & 0 & 0 & - & - & - & - \\
\hline & $\mathrm{AA}+\mathrm{GA}$ & 31 & 5 & 0.37 & 0.13 & 1.04 & 0.053 & 5 & 12 & 2.18 & 0.69 & 6.92 & 0.185 \\
\hline \multirow[t]{2}{*}{ rs10499194 } & $\mathrm{CC}$ & 94 & 37 & \multicolumn{4}{|c|}{ reference } & 31 & 42 & \multicolumn{4}{|c|}{ reference } \\
\hline & $\mathrm{CT}$ & 13 & 1 & 0.2 & 0.02 & 1.55 & 0.122 & 4 & 3 & 0.55 & 0.12 & 2.65 & 0.46 \\
\hline
\end{tabular}

The complement system may be abnormally increased and activated in RA [20]. We detected a lower C3 level among AA genotype individuals at rs13207033 compared with GG or GA patients ( $p=0.01$ for both). Therefore, GG and GA genotypes may be protective, whereas AA may pose a greater RA risk. A higher disease activity corresponds to suppression of the complement system, and vice versa. We found that $\mathrm{C} 3$ among ACA-positive patients was lower than among ACA-negative subjects. This may be due to an immune reaction between ACA and PLT phospholipids leading to complement activation and $\mathrm{C} 3$ consumption/decrease.

Though the specificity and sensitivity of the ESR are not ideal for the diagnosis of RA, it is considered a relatively reliable indicator of the acute phase reaction of inflammation. The CRP level is closely correlated with the degree of inflammation in RA. C-reactive protein is not only an inflammatory marker, but may also induce a proinflammatory effect by activating monocyte chemoattractant protein [21]. Yildirim et al. [19] and Zhang et al. [22] found that RA disease activity was positively correlated with the CRP level. Arvidson et al. [18] reported that FIB and CRP were tightly correlated to the activity of RA. This study found no difference in ESR or CRP across the TNF AIP3 SNPs examined.
Though the specificity of RF has little diagnostic significance for RA, it is an effective indicator of RA activity. A high titer of RF suggests a poor prognosis of RA [23]. We found that the RF-positive rate among the GG+TG patients at rs2230926 was lower than among the TT patients $(p=0.034)$. The TT genotype may be a risk factor for RA, whereas the GG+TG genotype may have a protective effect. The RF-positive rate among the TG patients was lower than among the TT patients $(p=0.051)$. The RF-positive rate among the GG+TG patients was even lower than among the TT patients, suggesting that the GG genotype may be a protective factor.

At present, the detection of anti-CCP antibody is one of the most specific tests for diagnosing RA. Most RA patients (70-80\%) harbor this antibody in their serum and synovial fluid during early stages of RA disease, even before the first symptoms appear [24-26]. Knoot et al. [27] reported that anti-CCP antibody was correlated with RA activity. Studies by Raptopoulou et al. [28] and Agrawal et al. [29] suggest that anti-CCP antibody can be used as a marker of RA activity. Glasnovic et al. [30] found that anti-CCP antibody could predict RA condition and that its titer was related to radiological joint lesions. However, anti-CCP antibody is not related to the joint tenderness index, the joint swelling 
index, morning stiffness, RF, CRP, or the ESR [31]. Although we did not detect a correlation between SNPs in rs13207033 and the occurrence of RA in our previous study, the anti-CCP antibody-positive rate among the AA+GA patients was lower than among the GG patients $(P=0.053)$, suggesting that the AA+GA genotype may be RA-protective, whereas the GG genotype may be a risk factor. The above result was indirectly supported by Viatte $\mathrm{S}$ et al. in anti-CCP antibody negative patients [32]. Recent results in Caucasian RA patients have demonstrated that the TNFAIP3 variant led to an increased risk of RA in a negative anti-CCP antibody and RF-positive patients compared with a positive anti-CCP antibody and RF negative patients [33, 34].

The presence of ACA can reflect the functionality of inflammatory endothelial cells and the tendency toward thrombosis, and some RA patients may harbor ACA. ACA cross-reacts with membrane phospholipids of endothelial cells and PLT as well as phospholipids in activated prothrombin complexes in the coagulation system; this causes increased phagocytosis and destruction of PLT by the reticuloendothelial system [35]. Though this study did not identify a correlation between TNFAIP3 SNPs and ACA.

In the Chinese Han population, TNFAIP3 SNPs are correlated with the following serological activity indicators of RA: the TT or TG genotypes at rs2230926 may be a risk factor indicated by IgG elevation, whereas GG may be a protective genotype; the TT genotype at rs2230926 may be a risk factor identifiable by RF positivity, whereas the GG+TG genotypes may be protective factors; GG or GA genotypes at rs13207033 may have protective effects and can be indicated by $\mathrm{C} 3$, whereas the AA genotype may be a risk factor; the AA+GA genotype at rs13207033 may be a protective factor and predicted by the level of anti-CCP antibody, whereas GG may be a risk factor.

In summary, the TT or TG genotypes at rs2230926 and the AA or GG genotypes at rs13207033 may be high-risk genotypes for RA as indicated by serological activity indicators in the Chinese Han population.

We are grateful to all subjects for their participation.

The authors declare no conflict of interests.

Conceived and designed the article: Xingang Zhang, Xiaofei Wang. Collected the cases and data acquisition: Xingang Zhang, Xiaoli Zhang. Molecular epidemiological studies, extraction and detection of DNA, detection of serological activity markers: Xingang Zhang, Li Jiang, Yun Guo. Sorted and analysed the data: Wei Li, Xinpeng Zhang. All authors read and approved the final manuscript.

\section{References}

1. Gregersen PK, Olsson LM (2009): Recent advances in the genetics of autoimmune disease. Annu Rev Immunol 27: 363-391.
2. Bowes J, Barton A (2009): Recent advances in the genetics of RA susceptibility. Rheumatology (Oxford) 47: 399-402.

3. Plenge RM, Cotsapas C, Davies L, et al. (2007): Two independent alleles at $6 \mathrm{q} 23$ associated with risk of rheumatoid arthritis. Nat Genet 39: 1477-1482.

4. Thomson W, Barton A, Ke X, et al. (2007): Rheumatoid arthritis association at 6q23. Nat Genet 39: 1431-1433.

5. Wertz IE, O'Rourke KM, Zhou H, et al. (2004): De-ubiquitination and ubiquitin ligase domains of A20 downregulate NF-kappaB signalling. Nature 430: 694-699.

6. Boone DL, Turer EE, Lee EG, et al. (2004): The ubiquitin-modifying enzyme A20 is required for termination of Toll-like receptor responses. Nat Immunol 5: 1052-1060.

7. Lee EG, Boone DL, Chai S, et al. (2000): Failure to regulate TNF-induced NF- $\kappa \mathrm{B}$ and cell death responses in A20-deficient mice. Science 289: 2350-2354.

8. Arnett FC, Edworthy SM, Bloch DA, et al. (1988): The American Rheumatism Association 1987 revised criteria for the classification of rheumatoid arthritis. Arthritis Rheum 31: 315-324.

9. Bottini N, Musumeci L, Alonso A, et al. (2004): A functional variant of lymphoid tyrosine phosphatase is associated with type 1 diabetes. Nat Genet 36: 337-338.

10. Begovich AB, Carlton VE, Honigberg LA, et al. (2004): A missense single nucleotide polymorphism in a gene encoding a protein Tyrosine phosphatase (PTPN22) is associated with rheumatoid arthritis. Am J Hum Genet 75: 330-337.

11. Orozco G, Reuda B, Robledo G, et al. (2007): Investigation of the IL-23R gene in a Spanish rheumatoid arthritis cohort. Hum Immunol 68: 681-684.

12. Lazarus M, Hajeer AH, Turner D, et al. (1997): Genetic variation in the interleukin 10 gene promoter and systemic lupus erythematosus. J Rheumato1 24: 2314-2317.

13. Sung YK, Park BL, Shin HD, et al. (2006): Interleukin-10 gene polymorphisms are associated with the SLICC/ACR Damage Index in systemic lupus erythematosus. Rheumatology (Oxford) 45: 400-404.

14. Wu RP, Hu YM, Jiang ZF (1996): Zhu Fu Tang Practical Pediatrics [M]. 6th ed. People's Medical Publishing House, Beijing: 1896-1898.

15. Andrews NC (2004): Anemia of inflammation: the cytokine-hepcidin link. J Clin Invest 113: 1251-1253.

16. Lee P, Peng H, Gelbart T, et al. (2005): Regulation of hepcidin transcription by interleukin-1 and interleukin-6. Proc Natl Acad Sci U S A 102: 1906-1910.

17. Jongen-Lavrencic M, Peeters HR, Vreugdenhil G, Swaak AJ (1995): Interaction of inflammatory cytokines and erythropoietin in iron metabolism and erythropoiesis in anemia of chronic disease. Clin Rheumatol 14: 519-525.

18. Johnson CS, Cook CA, Furmanski P (1990): In vivo suppression of erythropoiesis by tumor necrosis factor-alpha (TNF-alpha): reversal with exogenous erythropoietin (EPO). Exp Hematol 18: 109-113.

19. de Waal Malefyt R, Haanen J, Spits H, et al. (1991): Interleukin 10 (IL-10) and viral IL-10 strongly reduce antigen-specific human $\mathrm{T}$ cell proliferation by diminishing the antigen-presenting capacity of monocytes via downregulation of class II major histocompatibility complex expression. J Exp Med 174: 915-924.

20. Carroll MV, Sim RB (2011): Complement in health and disease. Adv Drug Deliv Rev 63: 965-975.

21. Yeh ET (2004): CRP as a mediator of disease. Circulation 109 (21 Suppl 1): II 11-14. 
22. Koury MJ (1992): Programmed cell death (apoptosis) in hematopoiesis. Exp Hematol 20: 391-394.

23. Tedesco A, D'Agostino D, Soriente I, et al. (2009): A new strategy for the early diagnosis of rheumatoid arthritis: a combined approach. Autoimmun Rev 8: 233-237.

24. Jansen AL, van der Horst-Bruinsma I, van Schaardenburg D, et al. (2011): Rheumatoid factor and antibodies to cyclic citrullinated Peptide differentiate rheumatoid arthritis from undifferentiated polyarthritis in patients with early arthritis. J Rheumatol 29: 2074-2076.

25. Liu FH, Zeng HL, Zheng XJ, et al. (2011): The significance of serum anti-CCP antibody, RF, CRP, ASO in the diagnosis of rheumatoid arthritis. J Clin Experimental Med 10: 583-585.

26. Chen Y, Pei B, Zu ML, et al. (2009): Study on the association of anti CCP antibody with disease activity in rheumatoid arthritis. J Clin Experimental Med 8: 9-10.

27. Kroot EJ, de Jong BA, van Leeuwen MA, et al. (2000): The prognostic value of anti-cyclic citrullinated peptide antibody in patients with recent onset rheumatoid arthritis. Arthritis Rheum 43: 1831-1835.

28. Kamradt T, Schubert D (2005): The role and clinical implications ofG6Pl in experimental models of rheumatoid arthritis. Arthritis Res Ther 7: 20-28.

29. Agrawal S, Misra R, Aggarwal A (2012): Autoantibodies in rheumatoid arthritis: association with severity of disease in established RA. Clin Rheumatol 26: 201-204.

30. Glasnovic M, Bosnjak I, Vcev A, et al. (2007): Anti-citrullinated antibodies, radiological joint damages and their correlations with disease activity score (DAS28). Coil Antropol 31: 345-348.

31. Cui HD, Zhang N, Fu HX, et al. (2006): The detection value of anti-cyclic citrullinated peptide antibody in patients with rheumatoid arthritis. Chinese J Practical Internal Med 26: 1977-1978.

32. Viatte S, Plant D, Bowes J, et al. (2012): Genetic markers of rheumatoid arthritis susceptibility in anti-citrullinated peptide antibody negative patients. Ann Rheum Dis 71: 1984-1990.

33. Perdigones N, Lamas JR, Vigo AG, et al. (2009): 6q23 polymorphisms in rheumatoid arthritis Spanish patients. Rheumatology (Oxford) 48: 618-621.

34. Patsopoulos NA, Ioannidis JP (2010): Susceptibility variants for rheumatoid arthritis in the TRAF1-C5 and 6q23 loci: a meta-analysis. Ann Rheum Dis 69: 561-566.

35. Schorer AE, Wickham NW, Watson KV (1989): Lupus anticoagulant induces a selective defect in thrombin-mediated endothelial prostacyclin releases and platelet aggregation. $\mathrm{Br}$ J Haematol 71: 399-407. 